Gastroenterologe 2013 $\cdot 8: 383-384$

DOI 10.1007/s11377-012-0739-x

Online publiziert: 3 . August 2013

(c) Springer-Verlag Berlin Heidelberg 2013

J. Keller ${ }^{1} \cdot$ P. Layer ${ }^{1} \cdot$ M. Fried ${ }^{2}$

${ }^{1}$ Medizinische Klinik, Israelitisches Krankenhaus, Hamburg

${ }^{2}$ Abt. Gastroenterologie und Hepatologie, UniversitätsSpital Zürich

\title{
Funktionelle Erkrankungen des Gastrointestinaltrakts
}

Die funktionellen Störungen des Gastrointestinaltrakts gehören wegen ihrer großen Verbreitung zu den häufigsten Krankheiten, die den Patienten zum Arzt führen. Dementsprechend machen sie einen großen Anteil in gastroenterologischen Praxen aus, etwa 30-50\% der Patienten. Für in der Klinik tätige Gastroenterologen spielen die meisten funktionellen Erkrankungen eine quantitativ kleinere Rolle, weil sie in der Regel ambulant diagnostiziert und therapiert werden können. Ein kleiner Teil der Patienten leidet aber unter heftigen, die Lebensqualität massiv verschlechternden Beschwerden, die dann sehr wohl zu stationären Aufenthalten führen. Überdies können diese Störungen mit den unterschiedlichsten abdominellen Symptomen sämtliche Abschnitte des Magen-Darm-Trakts betreffen, so dass sie, gerade auch wegen ihrer hohen Prävalenz, grundsätzlich als wichtige Differenzialdiagnose, aber auch als - das klinische Bild nicht selten exazerbierende bzw. verwischende - Begleitstörung anderer Erkrankungen in Betracht kommen.

Für Betroffene und betreuende Ärzte sind funktionelle gastrointestinale Störungen nach wie vor vielfach mit einem hohen Maß an Frustration verbunden, weil klinisch-apparative Untersuchungen definitionsgemäß unauffällig ausfallen und die Therapie häufig unklar oder wenig effizient ist. Dem Patienten mitzuteilen, alle Untersuchungen seien normal ausgefallen, er habe also „nichts“, demzufolge könne man auch „nichts“ tun, ist für die Führung des Patienten schädlich und entspricht auch nicht wissenschaftlichen Erkenntnissen.
Gerade die Pathophysiologie der wichtigsten funktionellen gastrointestinalen Erkrankungen, die funktionelle Dyspepsie und das Reizdarmsyndrom, wird heute wesentlich besser verstanden als noch vor wenigen Jahren. In einer Reihe von neueren, methodisch ausgezeichneten Studien konnten z. B. ausgeprägte mukosale Alterationen, insbesondere unterschwellige inflammatorische Prozesse nachgewiesen werden. Diese wiederum scheinen durch eine veränderte Freisetzung von Mediatoren enterische Neurone zu beeinflussen und können dadurch die schon lange bekannten gastrointestinalen Motilitäts- und Sensitivitätsstörungen dieser Patienten zumindest teilweise erklären. Parallel zu diesen grundlegenden Studien haben sich auch die diagnostischen und therapeutischen Konzepte erheblich verbessert.

Das vorliegende Schwerpunktthema in Der Gastroenterologe widmet sich dem wichtigen Themengebiet der funktionellen gastrointestinalen Störungen mit dem Ziel, Epidemiologie und Pathophysiologie sowie Diagnostik und Therapie der wichtigsten Erkrankungen auf dem Boden der aktuellen wissenschaftlichen Erkenntnisse und unter Berücksichtigung aktueller Leitlinien darzustellen. Es umfasst Artikel von international renommierten Experten zur funktionellen Dyspepsie einschließlich der funktionellen Ösophaguserkrankungen, zum Reizdarmsyndrom und zur chronischen Obstipation.

Die Ausführungen zur Epidemiologie sollen ermöglichen, die Relevanz der funktionellen Erkrankungen für die tägliche Praxis einzuschätzen. Die pathophy- 
siologischen Zusammenhänge werden ausführlich dargestellt, um dem betreuenden Arzt ein besseres Verständnis der Erkrankungen zu ermöglichen. Dies ist auch für die Patientenführung von Bedeutung. Pathophysiologische Kenntnisse sind die Voraussetzung für die Vermittlung eines tragfähigen Krankheitskonzepts, das dem Patienten seine Beschwerden angesichts der normalen Untersuchungsbefunde einleuchtend erklärt. Nur so kann der bei diesem Krankheitsbild häufige Wechsel der Ärzte mit nicht indizierten und teuren Wiederholungsuntersuchungen sowie therapeutischer Polypragmasie verhindert werden.

Die Abschnitte zur Diagnostik stützen sich unter anderem auf neuere Leitlinien und helfen dem Leser, eine gute Balance zwischen einer sorgfältigen Diagnosestellung mit Ausschluss von relevanten Erkrankungen und der Vermeidung einer ausufernden Diagnostik zu finden.

Abschließend werden die unterschiedlichen Therapieoptionen und deren wissenschaftliche Evidenz aufgezeigt. Schon aus der Vielzahl der aufgeführten therapeutischen Optionen wird deutlich, dass auch heute kein Standardmedikament zur Behandlung der funktionellen Dyspepsie, des Reizdarmsyndroms oder der chronischen Obstipation existiert. Stattdessen müssen häufig mehrere medikamentöse Optionen versucht werden, bevor eine adäquate Symptomlinderung beim individuellen Patienten erzielt werden kann. Auf der anderen Seite sind in den letzten Jahren mehrere neue Medikamente auf dem Boden aktueller pathophysiologischer Erkenntnisse entwickelt worden und es wird weiter nach neuen Therapieansätzen gesucht. Hierin liegt die Chance, dass die heute noch häufig ineffiziente Therapie der funktionellen gastrointestinalen Störungen in Zukunft wesentlich verbessert werden kann.

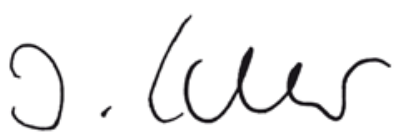

J. Keller

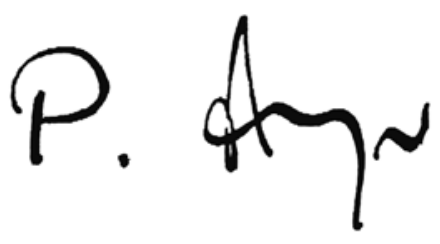

P. Layer

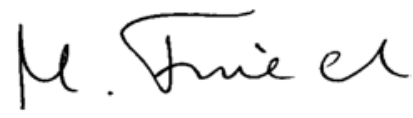

M. Fried

\section{Korrespondenzadressen}
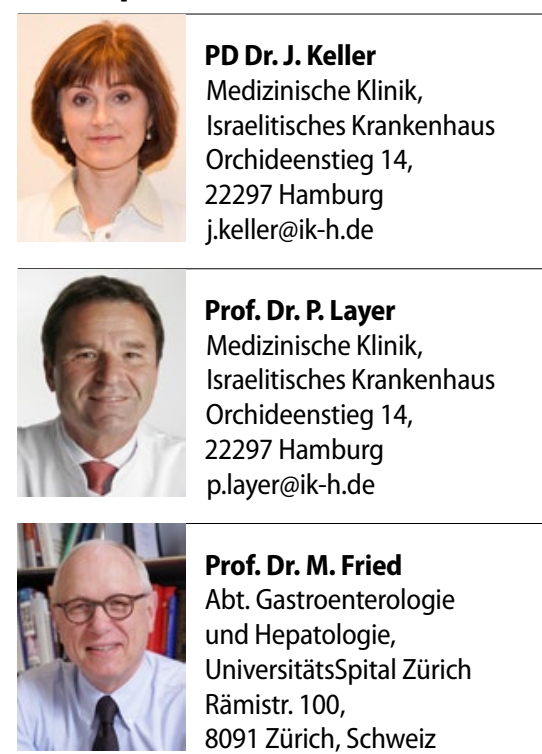

Prof. Dr. M. Fried

Abt. Gastroenterologie und Hepatologie, UniversitätsSpital Zürich Rämistr. 100, 8091 Zürich, Schweiz michael.fried@usz.ch

\section{Ärzte Zeitung digital ist Fachmedium des Jahres}

Digitale Formate sind auch in der medizinischen Fachpresse im Trend, und Springer Medizin setzt mit seinen Angeboten Maßstäbe. Die Fachverlagsgruppe hat für „Ärzte Zeitung digital", die App-Ausgabe der Tageszeitung, den renommierten Preis „Fachmedium des Jahres“ 2013 in der Kategorie „Bestes Mobiles Angebot" erhalten. Der Preis wird von der Deutschen Fachpresse in mehreren Branchen- und Sachkategorien verliehen. Die Deutsche Fachpresse ist die Marketing- und Dienstleistungsplattform für alle Anbieter von Fachinformationen im beruflichen Umfeld.

Mit dem Preis werden Publikationen gewürdigt, „'die beispielhaft für die vielen hochwertigen gedruckten und digitalen Informationsangebote aus Fachmedienhäusern in Deutschland stehen".

"Wir haben mit der "Ärzte Zeitung digital" konsequent unsere Tageszeitung für Ärzte in das digitale Zeitalter überführt", kommentiert Harm van Maanen, Executive Vice President von Springer Medizin, die Auszeichnung für die Fachtageszeitung. Die Strategie der Digitalisierung werde konsequent weiter verfolgt, so van Maanen weiter. So soll die App-Ausgabe, die bisher weitgehend die Inhalte der gedruckten Tageszeitung abbildet, eigenständig werden.

Die Ausgabe der „Ärzte Zeitung“ fürs iPad ist erstmals im November 2012 erschienen - im September 2013 folgt die Version für Android-Tablets. Das Angebot vervollständigt die digitalen Formate der Zeitung, die damit online über aerztezeitung.de, auf Smartphones (News App fürs iPhone sowie für Smartphones optimierte Website) und eben auch mit einer eigenen Ausgabe für Tablets erreichbar ist.

Quelle: Ärzte Zeitung 